\title{
The study on the quasi-single crystalline silicon ingot produced with plate seed block layer
}

\author{
Shuaidi Song ${ }^{1, a}$, Yang Cao ${ }^{1, b}$, Tingxi $\mathrm{Wu}^{1, \mathrm{c}}$ and Qiang Wang ${ }^{1, \mathrm{~d}^{*}}$ \\ ${ }^{1}$ School of Electronic and Information, Nantong University, Nantong 226019, China \\ a1007253629@qq.com,b2855912989@qq.com,c1140296861@qq.com,,wang_q@ntu.edu.cn
}

Keywords: quasi-single crystalline silicon ingot; crystalline growth; defects; silicon solar cell Abstract: The quasi-single crystalline silicon directional casting processes with ultrathin plate seed layers were studied. The experiments results showed that the directional casting with the plate seed layer could solve the cross pattern issue on the quasi-single crystalline silicon wafers produced with square seed blocks, and decrease the thickness of the plate seed blocks to $15 \mathrm{~mm}$. The ratio of single crystalline silicon in the quasi-single crystalline silicon ingot increased from $65 \%$ to $85 \%$ by extending the silicon melting time from 15 hours to 26hours and the crystalline growth time from 40hours to 76hours. The study on the defects in the ingot showed that the conversion efficiency of the quasi-single crystalline solar cells decreased with the defects increased from bottom to top in the ingot.

\section{Introduction}

The silicon solar cells have captured the most photovoltaic products market of the world due to its high efficiency and low production $\operatorname{cost}^{[1-2]}$. Crystalline silicon $(\mathrm{c}-\mathrm{Si}$ ) is the main substrate material for the manufacture of solar cells in the photovoltaic (PV) industry. In recent years, quasi-single crystalline (QSC) silicon has received much attention due to its high quality and low $\operatorname{cost}^{[3-4]}$.

The QSC silicon ingot directional casting is a new casting technology using the c-Si as the seed layer to obtain the QSC silicon ingots ${ }^{[5]}$. The QSC silicon ingots have the similar exterior and the electrical performance compared with the c-Si ingots ${ }^{[6-7]}$. The QSC casting method can be optimized by the process temperature to obtain the high quality and large single crystalline ratio QSC ingots ${ }^{[7-8]}$.

The QSC silicon ingots normally use the square c-Si as seed blocks which lead to the cross pattern issues on wafers and decrease the solar cells electrical performance ${ }^{[8-10]}$. The new plate seed blocks were used to produce the QSC silicon ingots which could efficiently solve the cross pattern issue on the wafers and enhance the electrical performances of the solar cells by the optimized crystalline growth processing parameters ${ }^{[11]}$.

\section{The $15 \mathrm{~mm}$ plate seed blocks bearing simulation}

The plate seed blocks were made of a c-Si ingot which sliced along the long axis. Six blocks of $980 \mathrm{~mm}$ long, $156 \mathrm{~mm}$ wide and $15 \mathrm{~mm}$ thick were chosen as the seed block to replace the square blocks. Due to the weight of the cubic loading reached $800 \mathrm{Kg}$, the plate seed blocks bearing were simulated to find out the plate blocks deformation.

\footnotetext{
*corresponding author: Qiang Wang
} 


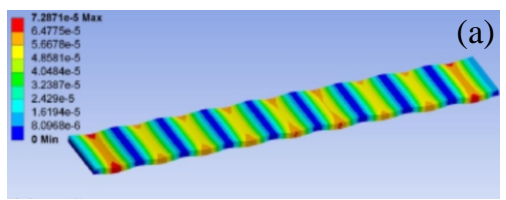

(a) interval

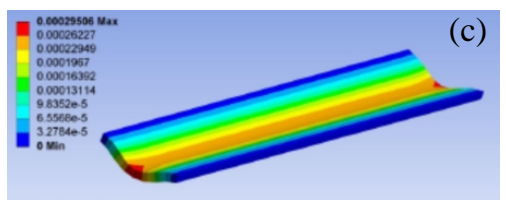

(c) long sides

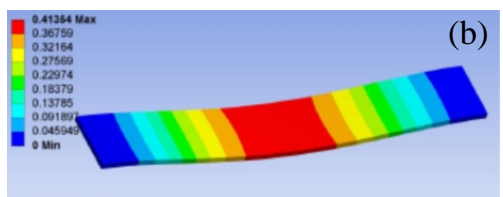

(b) short sides

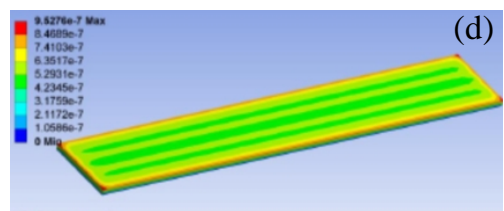

(d) full back

Figure 1 Plate seed block deformation simulation with four different bracing methods

(a) interval; (b) short sides; (c) long sides; (d) full back

Fig 1 showed four blocks bracing methods simulation results, (a) interval; (b) short sides; (c) long sides; (d) full back. Fig 1(a) showed that the plate seed block was supported every 100mm along the long side and the plate block deformation was $52 \mathrm{~nm}$ shaped like a wave. Fig 1(b) was the simulation result that short sides were braced and the plate block deformation was 400um. Fig 1(c) showed that the long sides support could result in $250 \mathrm{~nm}$ deformation. And the whole back support was almost no deformation showed in Fig 1(d). The simulation results showed that the $15 \mathrm{~mm}$ thickness plate seed block could bear the $800 \mathrm{Kg}$ loading.

\section{QSC silicon ingots directional casting experiments}

The QSC silicon ingot casting processes included: loading, evacuating, melting, crystalline growth, annealing and cooling, etc. Two casting experiments of different process time were done: 1) Evacuating the cavity lower than $0.008 \mathrm{mbar}$; 2) Heating for 15hours and 26hours, respectively, till temperature reaching $1540^{\circ} \mathrm{C}$ to completely melt silicon; 3) Raising the isolation cage to reduce the cavity temperature for crystalline growth and the process time was 40hours and 76hours, respectively; 4) Heating the ingot to $1085.5^{\circ} \mathrm{C}$ and annealing for $190 \mathrm{~min}$.

After the ingot casting process, 36 crystalline columns are obtained by slicing the ingot. The defect density and infrared scanning of the crystalline columns were studied by PL scanning and infrared detector respectively. The QSC solar cells were produced by Econess Energy Co., Ltd.

\section{Results and discussion}
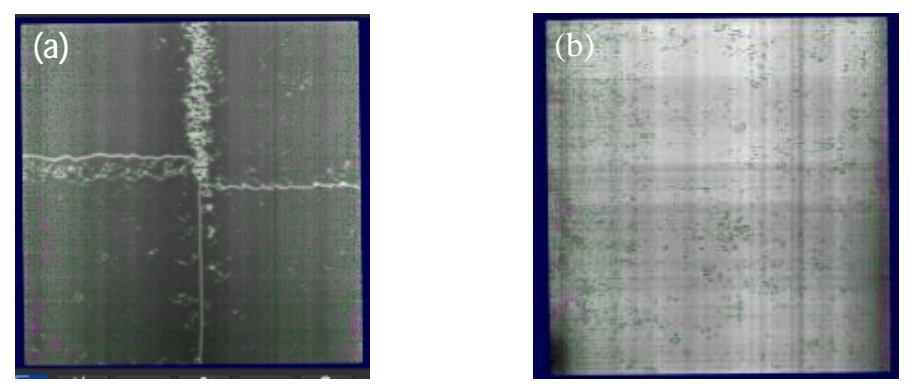

Figure 2 the PL image of wafers, (a) square blocks (b) plate blocks

Fig 2 showed the wafer PL image difference between the plate and square seed blocks. There was a cross pattern in the square seed block wafer, but none in the plate seed block wafer. A lot of defects were formed along the cross pattern which lead to the electrical performance decreasing due to the gaps between the square blocks. And the plate seed block wafers showed less defects than the square seed block wafers. 


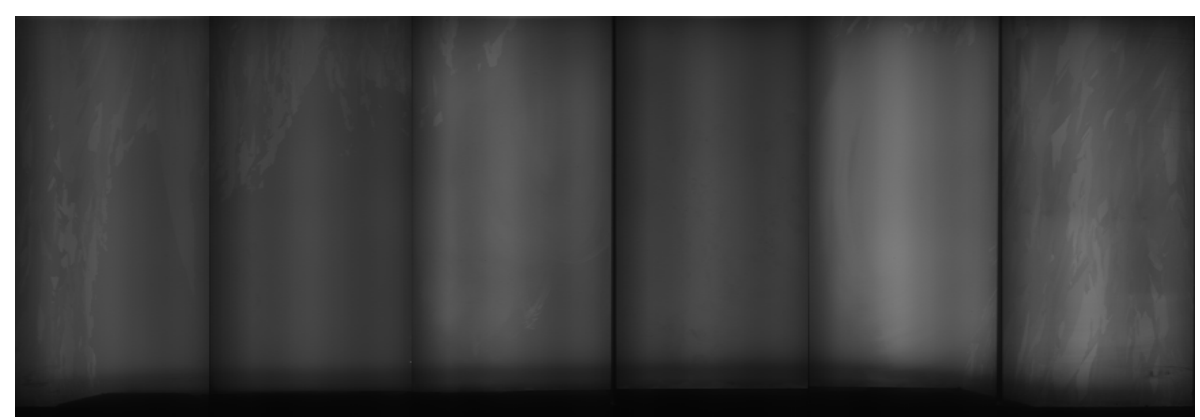

Figure 3 the infrared scanning image of QSC ingot with short process

Fig 3 showed the infrared scanning image of QSC ingot. From Fig 3 we could find that the ingot include single crystalline and multi-crystalline parts. The ratio of the single crystalline reached $65 \%$. The multi-crystalline part main located near the side wall of cubic due to near the heater ${ }^{[11]}$. And the seed blocks near the side wall of cubic were over melted, so the crystalline columns on them were multi-crystalline. The silicon melting time was 15 hours and this may cause the over melting phenomena because of too fast heating rate. There were some multi-crystalline on the top of the single crystalline columns which may be because the crystalline growth time was only 40hours and the process was also too fast.

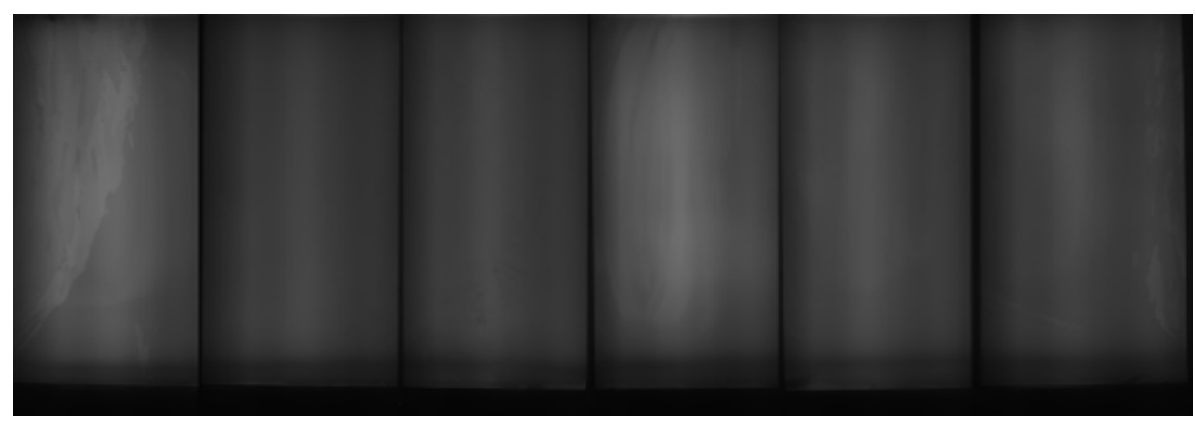

Figure 4 the infrared scanning image of QSC ingot with short process

Fig 4 showed the infrared scanning image of QSC ingot of 26hours melting and 76hours crystalline growth. The ratio of the single crystalline reaches $85 \%$. The rest of seed layer was strait and no place was over melted. Only part of the side crystalline columns was multi-crystallized. There was no multi-crystalline on top of the single crystalline columns. The extended melting and crystalline growth time could enhance the quality of the QSC ingots. Extending the melting time could reduce the heating rate which could efficiently avoid the over melting phenomenon. The longer crystalline growth time was, the slower crystalline grew. This provided enough time to form large single crystalline. The multi-crystalline near the cubic side wall was due to no seeding layer on the side wall.

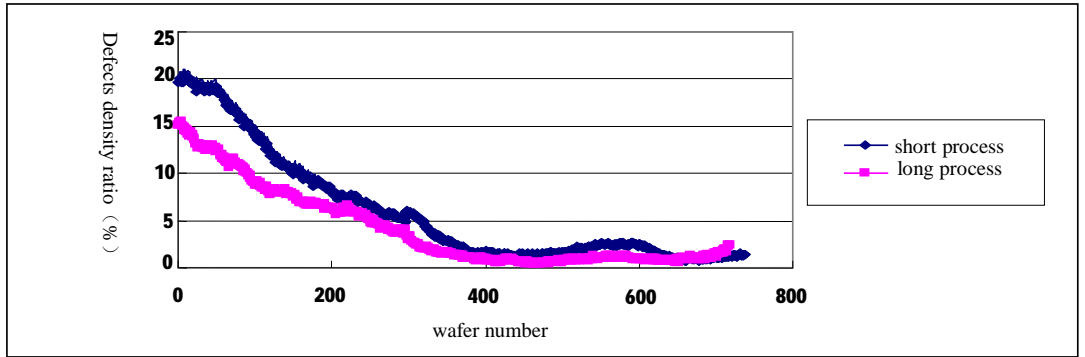

Figure 5 the defects density distribution in long (26hours melting, 76hours crystalline growth) and short processes (15hours melting, 40hours crystalline growth) ingots

Fig 5 showed the defects density in long and short processes ingots, respectively. The defects density distribution in the both ingots was similar. That was the defects density increased from 
bottom to top in the ingots. But the short process ingots showed higher defects density than the long process ingots. This meant that the long casting process provided enough energy for the silicon atom to form larger single crystalline. And this decreased the defects.

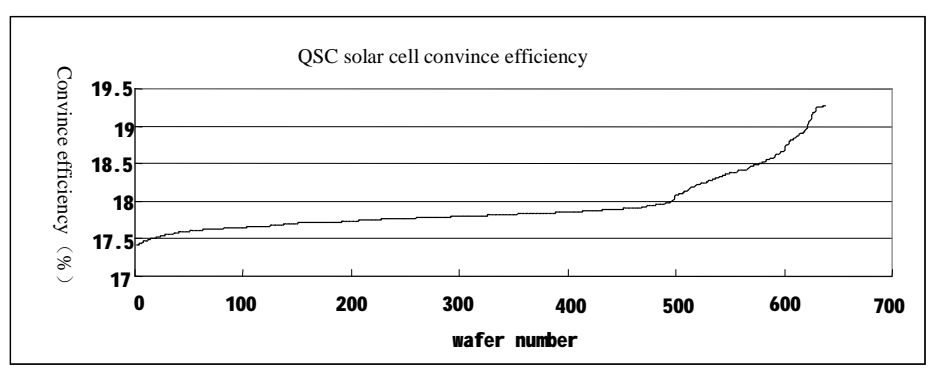

Figure 6 the QSC solar cell convince efficiency distribution from bottom to top of the ingot

Fig 6 showed the QSC solar cell convince efficiency distribution in the ingot. The solar cell convince efficiency decreased from bottom to top in the ingot. The highest convince efficiency of the solar cell was $19.3 \%$ at the bottom of the ingot which was much higher than multi-crystalline solar cells. The convince efficiency distribution was similar with the defect density distribution. This meant the defects were the key reason for QSC solar cell convince efficiency.

\section{Conclusion}

The QSC silicon ingot directional casting with plate seed blocks processes were studied. The wafers produced by plate seed block process showed no cross patterns. The single crystalline ratio in the ingot reached $85 \%$ which was much higher than the square seed blocks ingots. The process parameters were optimized that the melting time extending from 15hours to 26hours which could reduce the multi-crystalline ratio on the top of the ingot and the crystalline growth time extending from 40hours to 76 hours which raised the single crystalline ratio from $65 \%$ to $85 \%$. The defects density distribution increased from bottom to top in the ingots, while the convince efficiency decreased.

\section{Acknowledgments}

This work was financially supported by, Top-notch Academic Programs Project of Jiangsu Higher Education Institutions (PPZY2015B135), the Special Fund for the Transformation of S \&T Achievements of Jiangsu Province under Grant (BA2015045) and Jiangsu Foresight Project (BY2016053-10).

\section{References}

[1] Zhong GX, Yu QH, Huang XM, et al. Influencing factors on the formation of the low minority carrier lifetime zone at the bottom of seed-assisted cast ingots[J], Journal of Crystal Growth, 2014, 402: 65-70.

[2] Li ZY, Liu LJ, Zhou GS. Reusability of contaminated seed crystal for cast quasi single crystalline silicon ingots[J], Journal of Crystal Growth, 2015, 416: 159-163.

[3] Trempa M, Reimann C, Friedrich J, et al. Investigation of iron contamination of seed crystals and its impact on lifetime distribution in quasi-mono-crystalline silicon ingots[J], Journal of Crystal Growth, 2015, 429: 56-62.

[4] Tsoutsouva M.G., Oliveira V.A., Camel D., et al. Segregation precipitation and dislocation generation between seeds in directionally solidified mono like silicon for photovoltaic applications[J], Journal of Crystal Growth, 2014, 401: 397-403. 
[5] Gu X, Yu XG, Guo KX, et al. Seed assisted cast quasi single crystalline silicon for photovoltaic application Towards high efficiency and low cost silicon solar cells[J], Solar Energy Material and Solar Cells, 2012, 101: 95-101.

[6] Zhong GX, Yu QH, Huang XM, et al. Performance of solar cells fabricated from cast quasi single crystalline silicon ingots[J], Solar Energy, 2015, 111: 218-224.

[7] Wei Chen, Quanzhi Wang, Deren Yang, Lindong Li, Xuegong Yu, Lei Wang, Hao Jin. Influence of vertical temperature gradients on wafer quality and cell efficiency of Seed-assisted high-performance multi-crystalline silicon [J]. Journal of Crystal Growth, 2017, (467): 65-70

[8] Trempa M, Reimann C, Friedrich J, et al. Defect formation induced by seed joints during directional solidification of quasi mono crystalline silicon ingots[J], Journal of Crystal Growth, 2014, 405: 131-141.

[9] Tsoutsouva M.G., Oliveira V.A., Camel D., et al. Mono like silicon ingots grown on low angle misoriented seeds Defect characterization by synchrotron X-ray diffraction imaging[J], Acta Materialia, 2015, 88: 112-120.

[10]Gong LF, Wang FZ, Cai Q, et al. Characterization of defects in mono like silicon wafers and their effects on solar cell efficiency[J], Solar Energy Material and Solar Cells, 2014, 120: 289-294.

[11] Hu DL, Yuan S, He、 L, et al. Higher quality mono like cast silicon with induced grain boundaries[J], Solar Energy Material and Solar Cells, 2015, 140: 121-125. 